\title{
Development of Multi Channel Ad-Hoc Network System
}

\author{
Kentaro NISHIMORI $^{\dagger \text { a) }}$, Takefumi HIRAGURI ${ }^{\dagger \dagger}$, Masakatsu OGAWA ${ }^{\dagger \dagger}$, and Naoki HONMA ${ }^{\dagger \dagger \dagger}$, Members
}

\begin{abstract}
SUMMARY This paper proposes a new Ad-Hoc network system which comprises the multiple relay access points (APs) with multi channels. AdHoc network systems are recently proposed and incorporated for the communication infrastructure, which relays wireless transmission among access points (APs) in wireless LAN (WLAN) system. System throughput is decreased due to hidden terminal problem when only a single channel is used for the Ad-Hoc network. In order to solve this problem, a new system with multi channels is proposed. However, even if the multi channels are employed, the co- and/or adjacent-channel interference occurs due to hidden terminal problem and multiple APs in a limited space, when considering a simultaneous transmit and reception at the relay AP. In this paper, we develop an Ad-Hoc network testbed which can reduce and avoid coand/or adjacent-channel interference by using vertically arranged antenna configuration and distributed channel allocation scheme. Moreover, the effectiveness of our testbed is clarified by applying actual WLAN signals. key words: ad-hoc networks, vertical antenna arrangement, distributed channel allocation, multi channels
\end{abstract}

\section{Introduction}

Wireless LAN (WLAN) systems have been developed as access connections in order for wireless terminals to connect networks. WLAN services based on IEEE 802.11 standard [1] are now being used in home and office situations as well as many public places. Internet access via WLAN systems will be realized across extensive areas that cannot be served by wired network systems due to cost and geographic constraints. The studies [2]-[4] and standardization in IEEE 802.11s WG (Working group) [5] of mesh networks deals with the communication by the interconnection of WLAN, which achieves radio relay among two or more WLANs.

Moreover, IETF MANET (Mobile Ad-Hoc Networks) WG [6] is discussing the routing protocol for the communications among multiple wireless terminals. Ad-Hoc networks, which are temporarily configured by the multiple terminal stations, are standardized in IETF MANET WG. Wireless multi-hop using the WLAN is basically used for both the mesh network in IEEE802.11s and the Ad-Hoc network in MANET. The wireless multi-hop network is

\footnotetext{
Manuscript received June 15, 2010.

Manuscript revised October 30, 2010.

The author is with the Faculty of Engineering, Niigata University, Niigata-shi, 950-2102 Japan.

${ }^{\dagger \dagger}$ The author is with the Faculty of Engineering, Nippon Institute of Technology, Saitama-ken, 345-8501 Japan.

${ }^{\dagger \dagger}$ The author is with Technical Assistance and Support Center, NTT East Corporation, Tokyo, 144-0053 Japan.

${ }^{+1+\dagger}$ The author is with the Faculty of Engineering, Iwate University, Morioka-shi, 020-8551 Japan.

a)E-mail: nishimori@m.ieice.org

DOI: 10.1587/transcom.E94.B.667
}

providing the chance of the communication for the wireless terminals which are placed very far away from each other, even when they cannot communicate in direct link. Hence, WLAN has been recently paid much attention. With using Ad-Hoc systems, the infrastructure built by some APs will be one of the bearer networks that are available anytime-anywhere. Therefore, Ad-Hoc network techniques are expected to provide new multimedia applications, such as the video delivery to wide area or dead zone, since that can create temporary networks for disastrous area, and inter-vehicles communication in intelligent transport system (ITS), etc.

Multi channel systems are proposed on Ad-Hoc networks using the WLAN, in order to avoid interference due to hidden terminal problem [7]-[11] and increase system throughput. Although such studies are based on the theoretical evaluation using computer simulation, there are some issues for achieving the multi-hop system in the actual device and system. One of the problems is the adjacent channel interference. Even if the multi-hop system operates different channels, the transmitting signal of the other channel affects the adjacent channels due to its leakage power among the devices which are installed closely together inside the common aluminum chassis. Hence, the throughput performance by the relay transmission is degraded by such an interference [12].

There is another issue to realize the multi channel systems. When the number of terminals is increased while the available frequency bands are limited, the system throughput is decreased by co-channel interference if the system employs careless channel number selection. It is reported that the system throughput is decreased due to hidden terminal problem in wireless multi-hop network even if the multi frequency channels are used [13]-[15]. However, countermeasures relating to these studies are complex to implement and an extensive modification is required for IEEE802.11 standard. Therefore a simple channel allocation schemes should be implemented in order to develop a hardware testbed for avoiding the co-channel interference due to the hidden terminal problem.

In this paper, the Ad-Hoc network system, which comprises the multiple relay access points (APs), is proposed and implemented by an actual testbed. In the testbed there are two proposals regarding antenna configuration of the AP and channel allocation scheme for Ad-Hoc network system using multi channels. Furthermore, two proposed techniques are implemented on the testbed and the effectiveness 
of the proposed techniques is confirmed by throughput experiments using actual WLAN signals.

The remainder of this paper is organized as follows. In Sect. 2, we describe the target and issue on multi channel systems. Section 3 proposes the interference suppression using a vertical array antenna. In Sect. 4 , we propose the channel allocation scheme. The effectiveness of the testbed, in which the proposed schemes are implemented, is shown using actual WLAN signals in Sect. 4.

\section{Target Scenario and Problem}

The network configuration of the Ad-Hoc network system comprises the multiple relay access points (APs) with multi channels as shown in Fig. 1. $L_{N}$ denotes a wireless relay link and $N$ is the number of links in Fig. 1. There is a possibility that each AP links to neighboring APs dynamically in multi-hop relay access system with multi channel access. To realize this network configuration, a relay AP must be able to transmit and receive the signals at the same time using the different frequency channels. Figure 2 shows a configuration of the developed AP which can realize the multi-hop network system with multi channel access. The specification of developed AP is shown in Table 1. This AP has four WLAN devices, and these devices control communication link and data transfer, independently.

The main function of MAC protocols is Carrier Sense Multiple Access with Collision Avoidance (CSMA/CA),

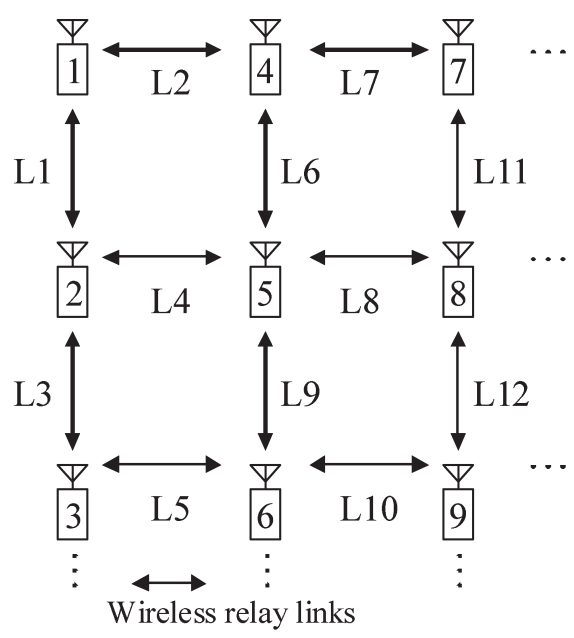

Fig. 1 Configuration of Ad-Hoc network system.

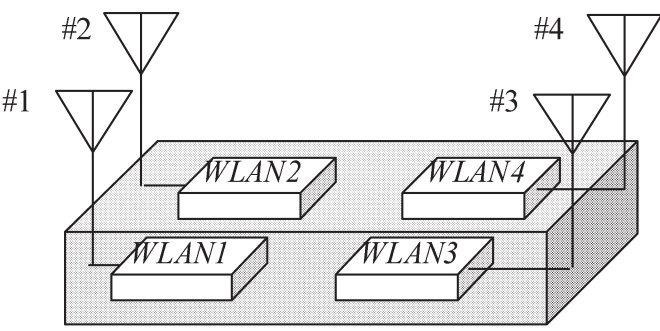

Fig. 2 Configuration of multiple APs with multi channels. which is implemented in WLAN system. CSMA/CA attempts to prevent a terminal from transmitting at the same time as other terminals within the same transmitting range by listening to the signals within the same channel before transmitting on each terminal. Therefore, CSMA/CA is useful function for a single cell situation, where same channel is shared by the terminals. However, since the distance between APs will be far rather than a single cell in the Ad-Hoc network, the hidden terminal problem occurs as shown in Fig. 3, since the signal of the AP1.cannot reach AP2, and vice versa. The most famous scheme uses frame exchange with Request-To-Send (RTS) and Clear-ToSend (CTS) frames (Two-way handshake) in order to eliminate the interference between hidden terminals [1]. However, since RTS/CTS have large overhead, throughput performance is degraded. Moreover, the interference occurs, if RTS frames are sent from multiple terminals at the same time. Therefore, in order to avoid this issue, multi channels are utilized in the developed system.

Figure 4 shows an example for two kinds of interference in multi-hop network system with multi channels. For

Table 1 Specification of developed AP.

\begin{tabular}{|c|l|}
\hline Item & Specifications \\
\hline WLAN & $\begin{array}{l}\text { Number of WLAN device: } 4 \\
\text { WLAN IEEE802.11b/g }(2.4 \mathrm{GHz} \text { band })\end{array}$ \\
\hline Ethernet & $\begin{array}{l}\text { Number of interfaces: } 1 \\
10 / 100 \text { Base-T }\end{array}$ \\
\hline CPU & SH4 \\
\hline OS & Linux Kernel 2.6 \\
\hline RAM & DDR-SDRAM: 64 MByte \\
\hline Chassis & Aluminum $230 \times 210 \times 60 \mathrm{~mm}$ \\
\hline Weight & $500 \mathrm{~g}$ \\
\hline
\end{tabular}
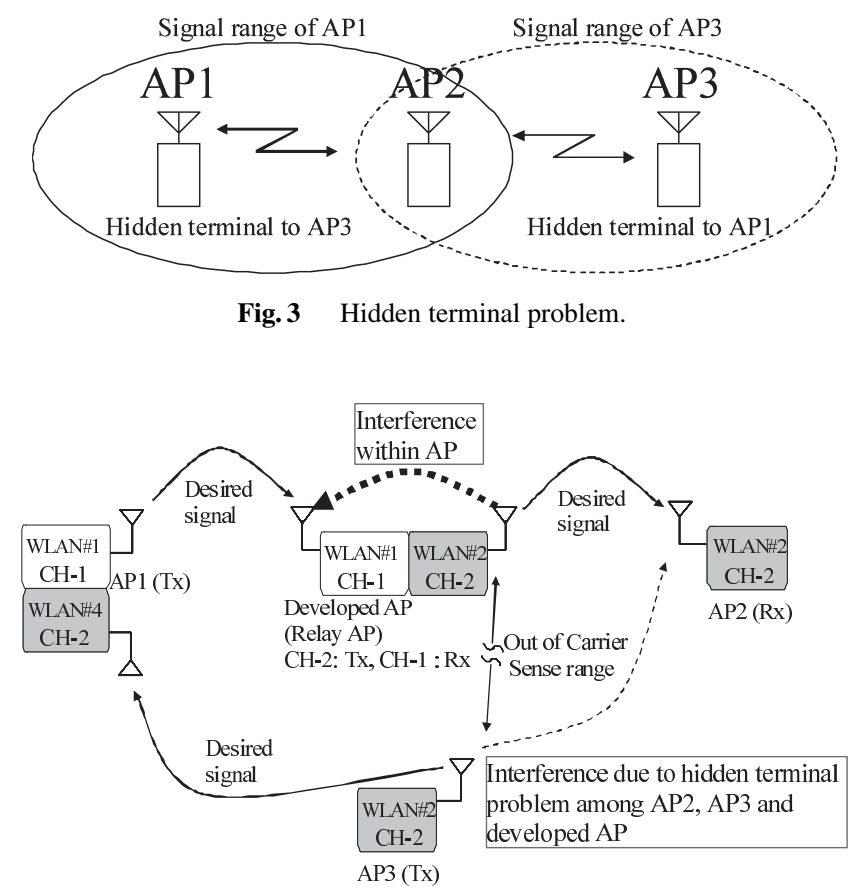

Fig. 4 Two kinds of interferences. 
simplification, we assume that the developed AP uses the channel 1 (CH-1) and channel $2(\mathrm{CH}-2)$ for receiving and transmitting signals, respectively, in Fig. 4. As a first interference, the interference due to hidden terminal problem is considered. As shown in Fig. 4, when the AP3 tries to transmit the signals to the AP1 with $\mathrm{CH}-2$, the interference from the AP3, which uses the same frequency channel with the developed AP, also arrives at the AP2. The hidden terminal problem occurs as shown in Fig. 3, because the AP2 receives the signal from the AP3 while the developed AP cannot recognize the AP3 due to the obstacle such as buildings and out of range on the carrier sense as shown in Figs. 3 and 4.

The second problem is that the interference due to a leakage power among devices, which are implemented inside same aluminum chassis. As shown in Fig. 4, the developed AP tries to communicate with AP1 and AP2 at the same time using $\mathrm{CH}-1$ and $\mathrm{CH}-2$, respectively. Even if the adjacent channels are used among WLAN devices, the interference from $\mathrm{CH}-2$ arrives at the spurious on the $\mathrm{CH}-1$, because the received power between the transmitter and receiver at the developed AP is much higher than the receiver at the developed AP from the AP1. It is reported that the mutual interference among adjacent channels is a big problem [12], when WLAN devices are mounted in adjacent space and each device use neighbor channels.

In this paper, we propose two techniques in order to solve two kinds of interference problems. One of the proposed techniques is the interference suppression using the null direction of the radiation pattern of the dipole antenna. The proposed antenna can reduce the mutual interference inside the developed AP by arranging dipole antenna vertically since this direction corresponds to the null direction of the radiation pattern. Second proposal is relating to distributed channel allocation scheme. The second proposal recognizes simple network topology and allocates channels most suitable for Ad-Hoc network, in order to avoid the interference due to the hidden terminal problem. Moreover, the proposed two techniques are implemented in the developed AP. We introduce the testbed and clarify the effectiveness of the testbed.

In the conventional technology implemented on testbed [20], the destination AP was specified by using the directional antennas, and the routing paths were constructed. In addition, the channel allocation scheme uses an algorithm that considers the directional antenna. For the feature of proposed techniques in this paper, multi-antennas are also used to relay communication, but the omni directional antennas are adopted instead of directional antennas. These omni directional antennas are utilized to decrease the interference between antennas for two combined APs. Furthermore, a complex algorithm is not required even when the relay APs are are simply arranged without taking care of the interference, and the wireless relay systems/Ad-Hoc systems can be achieved simply. Therefore, the proposed channel allocation is suitable for APs with omni directional antennas.

\section{Proposed Antenna Configuration}

\subsection{Principle}

In this section, we describe the requirement of AP antenna and propose a novel antenna configuration which can efficiently suppress the mutual interference inside the developed AP. The objective of this study is developing the AP system suitable for the Ad-Hoc relay system. For this system, the antenna pattern in the horizontal plane should be omni-directional because the relay station must transmit/receive signal to/from all of directions. Another requirement is that the antenna must suppress the interference among jointly placed APs while allowing APs to receive sig-
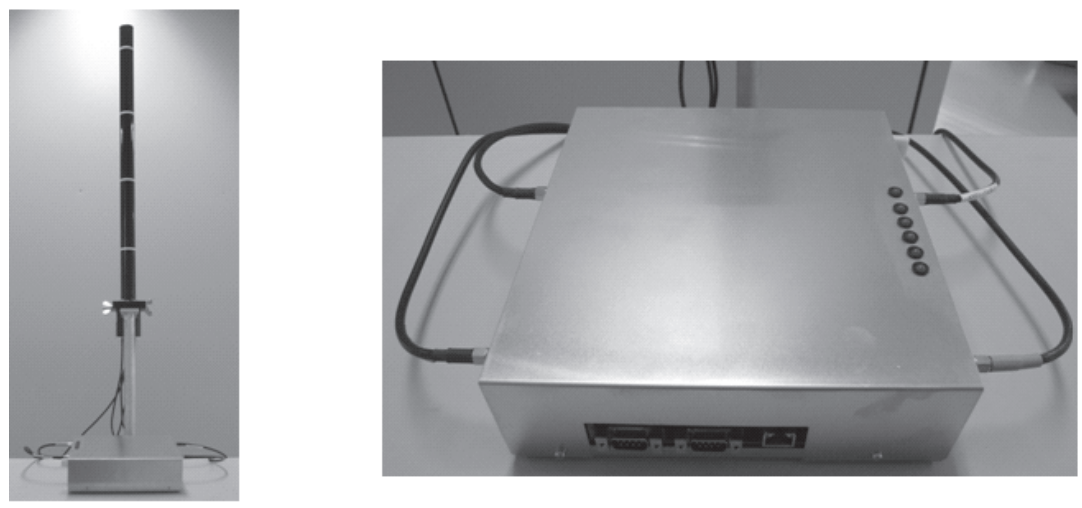

(a) Whole picture

(b) Chassis

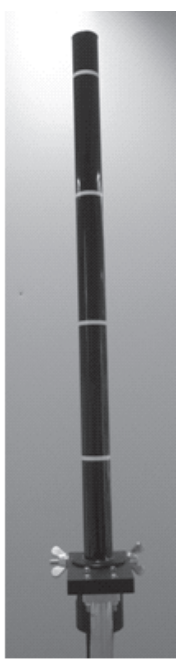

(c) Antenna

Fig. 5 Photo of developed AP. 


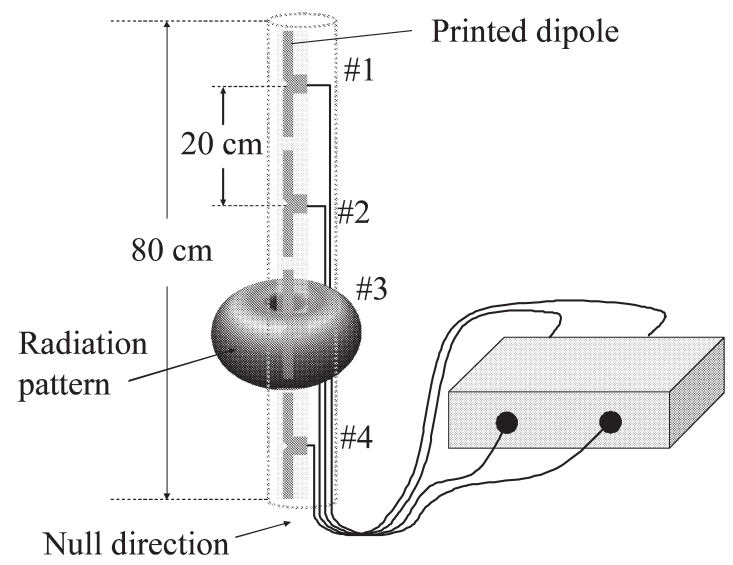

Fig. 6 Proposed antenna configuration using vertically deployed dipole antennas.

nals from separated APs.

Figure 5 is the photograph of the developed AP. Four antennas are configured inside of a cylinder. The vertically arranged dipole array is introduced to the AP system while the horizontally spaced dipole array is equipped in Fig. 3. The other functions are designed based on the concept shown in Fig. 3. Four frequency channels on IEEE $802.11 \mathrm{~b} / \mathrm{g}$ $(1,6,11,14 \mathrm{ch})$ are assigned on the testbed. Figure 6 reveals the antenna geometry of the proposed configuration in detail. The distance between neighboring antennas is $20 \mathrm{~cm}$, and the total length of the array antenna is $80 \mathrm{~cm}$. Four vertically polarized dipole antennas are arranged along the null direction of their radiation pattern. This arrangement provides high isolation among the antennas even when they are placed closely each other since no radiation power is delivered to neighboring antennas.

Other important feature of this antenna is that all antennas have omni-directional patterns in the horizontal plane. This property satisfies the requirement that AP must be able to communicate with other separated APs. All antennas are printed dipoles and configured on the common substrate. Each printed dipole has an independent feedline, which is connected to sole AP module inside the chassis. Conventional array antenna using dipole antenna in the vertical plane is called as co-linear array. However, those ports are connected with one another in order to receive/transmit signals on the conventional co-linear array, because the colinear array is used in order to obtain the higher antenna gain compared to the single dipole antenna. On the other hand, each dipole antenna in the proposed system is used for the mutual interference suppression among antennas in the vertical plane. This configuration achieves high interference suppression between adjacent channels and enables arbitrary AP in the system to transmit the signals while the other one is receiving the signal from another STA. As described above, this antenna configuration satisfies both two requirements: 1) to be able to suppress the interference among the jointly placed APs, and 2) to be able to communicate with other separated APs.

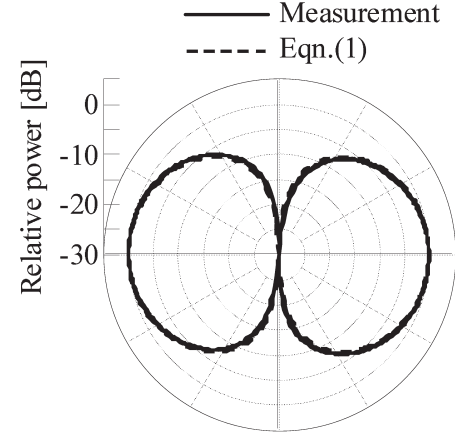

Fig. 7 Radiation pattern of dipole antennas on vertical plane.

Adaptive array antenna is proposed to reduce the cochannel interference [16]. The adaptive array supresses the interference by using antenna arrays and signal processing techniques. Moreover, the interference cancellation techniques are proposed using the array antenna which is arranged in the vertical plane [17], [18]. However, as mentioned above, the proposed antenna configuration utilizes the feature on the antenna pattern which is arranged in the vertical plane for each antenna: There is no signal processing unlike the adaptive array. Figure 7 shows the comparison between the calculated and measured antenna patterns in the vertical plane. The radiation pattern $F$ of the halfwavelength dipole antenna in the vertical plane is shown as follows:

$$
F(\theta)=\left|\frac{\cos \left[\frac{\pi}{2} \cos (\theta)\right]}{\sin (\theta)}\right|
$$

where $\theta$ denotes the angle in Fig. 7. As can be seen in Fig. 7, the measured radiation pattern agrees with the calculated pattern well. Moreover, we found that, the null over $-30 \mathrm{~dB}$ can be created for the directions on the adjacent antennas.

\subsection{Effectiveness of Proposed Antenna Configuration}

We confirm the effectiveness of proposed antenna configuration by comparing the conventional array antenna whose antenna is arranged in the horizontal plane. Figure 8 shows evaluation scheme. $\mathrm{AP}_{B}$ is the $\mathrm{AP}$ on conventional or proposed system and $\mathrm{AP}_{A}$ and $\mathrm{AP}_{C}$ are $\mathrm{APs}$ which are used for this evaluation. The measurements are carried out in an anechoic chamber, because the effect on sole antenna is evaluated. The signal $(1 \mathrm{ch})$ from $\mathrm{AP}_{A}$ is received at $\mathrm{AP}_{B}$ and the signal $(14 \mathrm{ch})$ is transmitted from $\mathrm{AP}_{B}$ to $\mathrm{AP}_{C}$. For the proposed configuration, the element spacing, $d$ is fixed to be $20 \mathrm{~cm}$ and vertically arranged dipole array as shown in Fig. 6 is used. The element spacing, $d$ is changed from 20 to $200 \mathrm{~cm}$ in the conventional configuration (horizontally arranged dipole array).

Figure 9 shows the measurement results of the average throughput versus the element spacing, $d$. As can be seen in Fig. 9, the throughput without the interference (6.7 Mbps) is achieved when the proposed antenna configuration is used. 
On the other hand, $d$ must be separated $120 \mathrm{~cm}$ in the conventional antenna arrangement.

We focus on the mutual coupling level between two antennas in Fig. 10. As can be seen in Fig. 10, the array antenna with the vertical arrangement realizes the mutual coupling reduction of over $20 \mathrm{~dB}$ compared to the array antenna
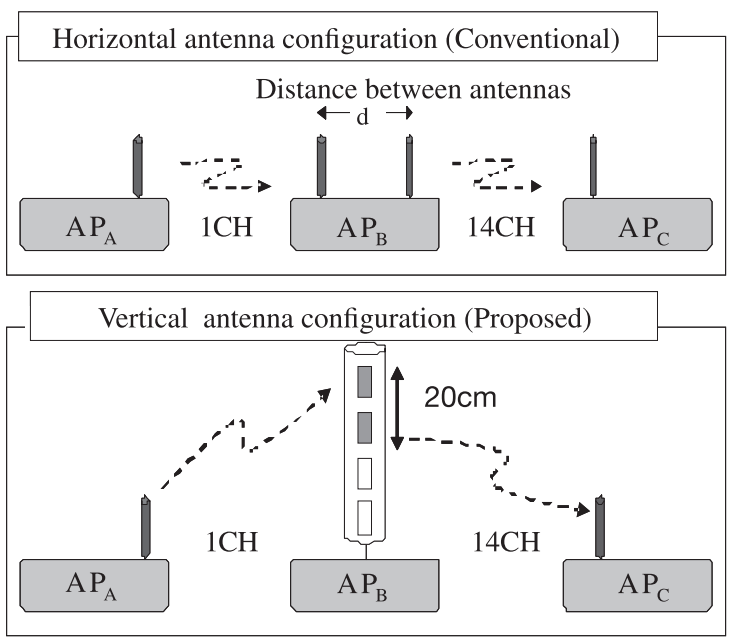

Fig. 8 Configurations for evaluation.

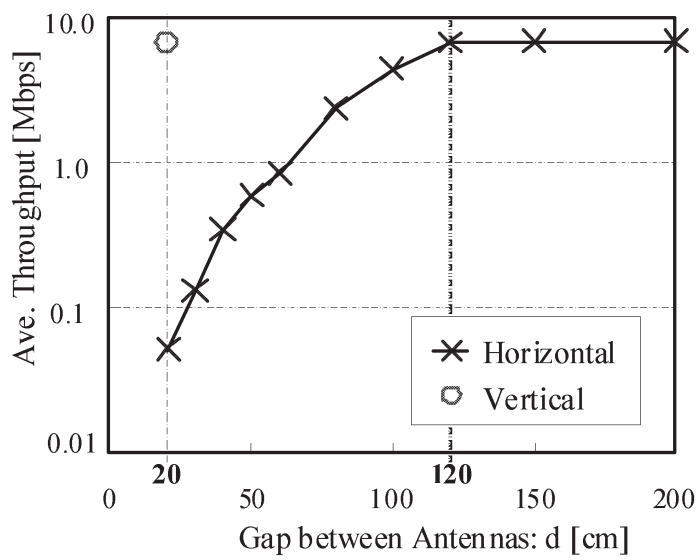

Fig. 9 Throughput of UDP flow.

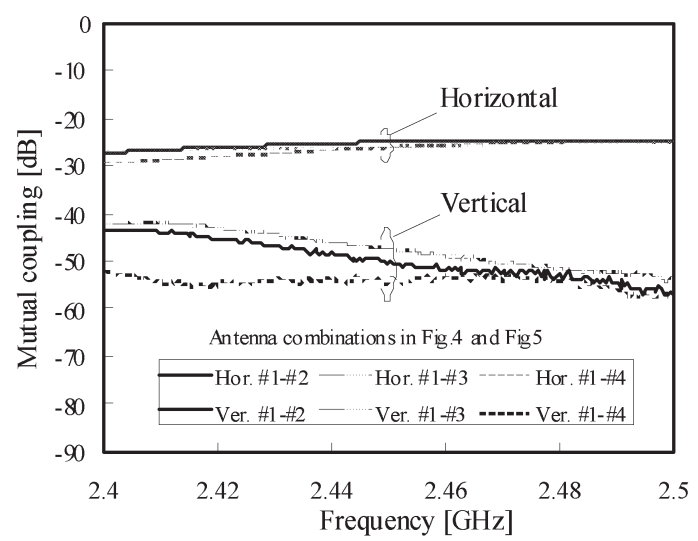

Fig. 10 Mutual coupling between antennas. with the horizontal arrangement. Hence, we found that the antenna configuration with vertical antenna arrangement is effective in reducing the mutual interference power with fair array antenna size.

\section{Channel Allocation Scheme}

It is essential to mitigate the interference from hidden APs in a multi-channel configuration as it is in a single-channel configuration. Channel-allocation schemes play an important role in mitigating hidden APs. Although there have been numerous studies on channel allocation [19], actual performance has not been evaluated with an actual testbed in any of these.

The hidden terminal problem was not evaluated sufficiently in [19], because they did not properly describe how the network topology was configured. However, because of the effect of the antennas we propose, our new ad-hoc network system can disregard interference between antennas and it simply allocates the best channel. Moreover, since the purpose of the proposed channel-allocation scheme is to avoid the hidden-terminal problem, the purpose of this paper is completely different from the work in [19]. Finally, while we understand the importance of a channel-allocation scheme for routing that suits all applications and maintains the quality of links (transmission rate and delay), studies on these issues will remain to be done in future work because their purpose regarding these issues was different from that dealt in this paper.

It is important to implement a channel-allocation scheme on a testbed and clarify how effective the implemented scheme is to confirm its behavior. Since the ad-hoc network is built in autonomously in a distributed manner, we focused on an autonomous-distributed scheme for allocating channels that used localized information.

There is a brief overview of the channel-allocation scheme we developed in Fig. 11. As can be seen from this figure, we assumed that the network consisted of nine APs that were deployed in a grid pattern. In addition, the range of communications for each AP reaches adjacent APs. The point of this scheme was to allocate channels by taking into

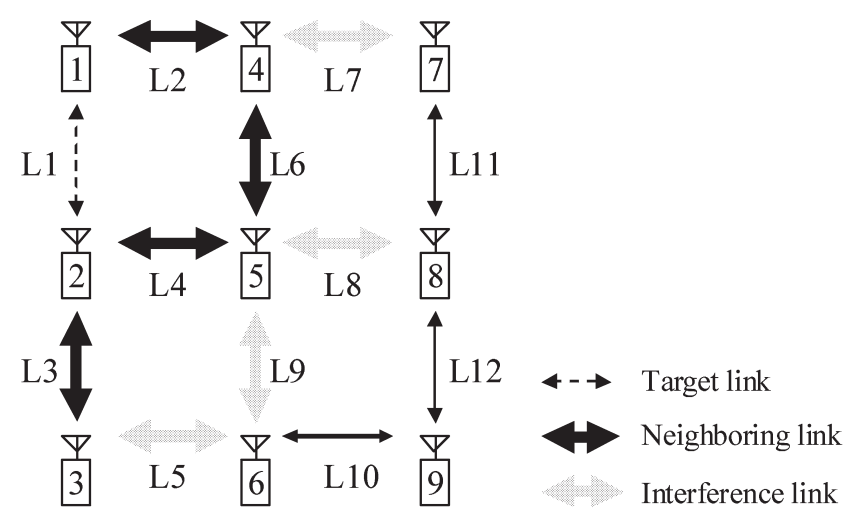

Fig. 11 Concept underlying method of channel allocation. 
consideration the effect from interfering links ${ }^{\dagger}$. For example, when the channel on link 1 (L1) is allocated as shown in Fig. 11, the channels on its links (L5, L7, L8, and L9) interfering with L1 have to be taken into account. That is, an interfering link means a link of hidden APs (AP6, AP7 and AP8) interfering with APs (AP1 and AP2) on the target link. Basically, the target link (L1) is allocated a channel that is different from the channel of its interfering links (L5, L7, L8, and L9).

The channel-allocation scheme we developed involves four operrations:

(1) Detection of neighboring APs.

(2) Recognition of network topology.

(3) Selection of AP for establishing links.

(4) Algorithm for allocating channels.

Each AP has a unique ID (APID) to autonomously allocate channels and they exchange information concerning channels that are allocated. When an AP is initially deployed, it detects its neighboring APs. After the AP has collected the information for allocating channels from neighboring APs, it recognizes the network topology around itself. Then, the AP selects an AP from APs to establish a link. Finally, the AP allocates a channel for the link with the channelallocation algorithm, which uses the information from interfering links. As stated above, the proposed scheme is distributed autonomously and is scalable because the scheme only uses local information around a newly deployed AP. We developed a channel allocation module operated in the MAC layer to accomplish a channel-allocation scheme in actual devices. We used the MAC layer functions provided by the software design kit (SDK) to establish the number of channels for all WLAN devices.

\subsection{Neighboring AP Detection}

A newly deployed AP broadcasts an AP information request on a common channel to detect its neighboring APs. APs that receive the $A P$ information request reply with their $A P$ information response. The newly deployed AP recognizes its neighboring APs after it receives their AP information responses. An AP information request consists of a source APID. An AP information response consists of a destination APID, a source APID, AP information (neighboring APIDs, channel number, and number of hops from the gateway), and neighboring AP information (APID and the channel numbers for all WLAN devices). In this way, the newly deployed AP collects the local information around itself.

\subsection{Network Topology Recognition}

After the newly deployed AP has collected the AP information responses from its neighboring APs, it recognizes the network topology so that it can allocate channels. For example, a newly deployed AP (AP1) detects the AP2 in Fig. 11. To allocate a channel on L1, AP1 has to recognize channels with interfering links (L5, L7, L8, and L9). As seen in Fig. 12, AP1 recognizes the network topology from the channel numbers and links in that information by receiving AP information responses from AP2 and AP4.

More specifically, after AP1 has received an AP information response from $\mathrm{AP} 2$, it obtains information that is from AP2's neighboring APs (AP3 and AP5), channel numbers connected to neighboring APs, and channel numbers that are used in neighboring APs. Then, after AP1 has received an AP information response from AP4, it obtains information that is from AP4's neighboring APs (AP5 and AP7), channel numbers connected to neighboring APs, and channel numbers that are used in neighboring APs. Finally, AP1 recognizes the network topology around itself, as shown in Fig. 13. This channel-allocation scheme only uses a localized network topology and does not use the entire network topology.

\subsection{Selection of AP for Establishing Link}

The newly deployed AP recognizes the number of hops from the gateway to all neighboring APs by receiving an AP information response from its neighboring APs. It then selects an AP from the APs to establish a link with two rules. 1) The received signal strength indicator (RSSI) is greater than a pre-defined value and 2) the smallest number of hops from the gateway. A neighboring AP satisfying these two conditions is selected. In other words, these rules mean the link is stable and it has the shortest path to the gateway. RSSI in the MAC frame is aggregated (desired and undesired) signals.

\subsection{Channel-Allocation Algorithm}

The newly deployed AP recognizes the network topology around itself and it selects an AP from neighboring APs to establish the link according to the procedure described in the previous subsections. Finally, a channel on the link is allocated. The policy in this algorithm is to mitigate interference from hidden APs. For the sake of simplicity, this subsection explains how a newly deployed AP establishes links for all of its neighboring APs without taking into consideration the number of hops from the gateway. By recognizing the network topology described in Sect. 4.2, the newly deployed AP knows the channels on interfering links. If more than one available channel differs from the one that is present, the newly deployed AP selects one of the available channels.

However, if no available channel is present, the newly deployed AP selects the channel with the smallest RSSI. Let us more specifically explain the channel-allocation algorithm using the target link (L1) in Fig. 13. The available channels are initially $\mathrm{CH} 1, \mathrm{CH} 2, \mathrm{CH} 3$, and $\mathrm{CH} 4$. After the newly deployed AP (AP1) receives an AP information response, it knows $\mathrm{CH} 2, \mathrm{CH} 3$, and $\mathrm{CH} 4$ are used in interfering links. Here, since the available channel is $\mathrm{CH} 1$, which is different from the channels on interfering links $(\mathrm{CH} 2, \mathrm{CH} 3$,

${ }^{\dagger}$ Interfering links mean those between hidden APs and the neighboring APs of APs on a target link. 


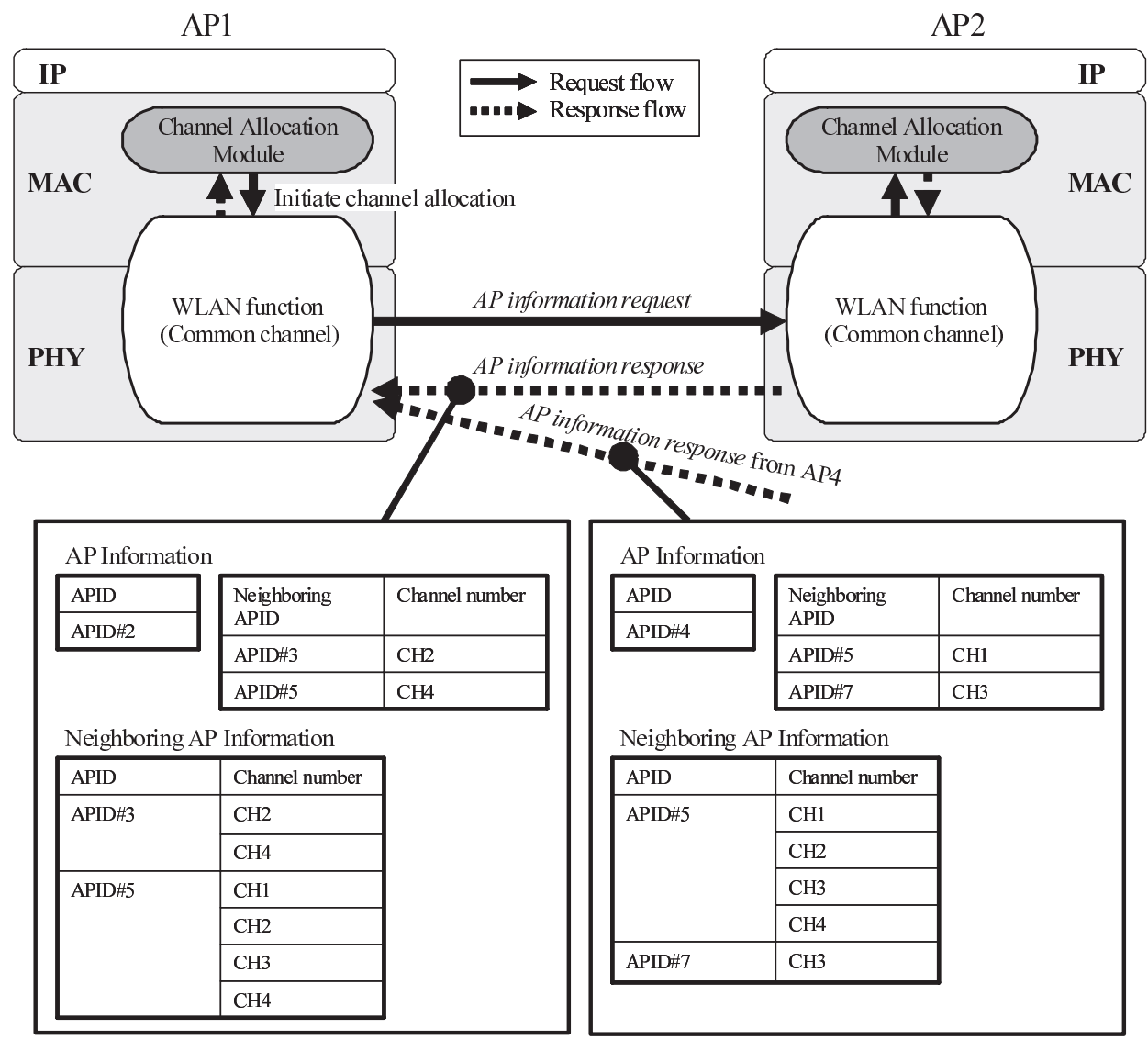

Fig. 12 Example of AP information response.

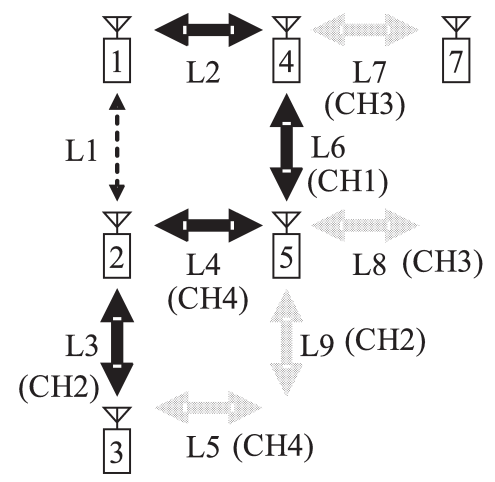

Fig. 13 Recognized network topology at AP1.

and $\mathrm{CH} 4)$, AP1 allocates $\mathrm{CH} 1$ to $\mathrm{L} 1$, which connects to AP2.

\subsection{Evaluation of Performance with Channel Allocation}

How effective the testbed was with the proposed schemes is clarified in this subsection by comparing throughput between single and multi-channels using WLAN signals. Figure 14 shows the configuration for the measurement system. We employed the setup for allocating channels and measuring throughput using the five APs in Fig. 14. The standard for the WLAN was IEEE802.11g and the common channel

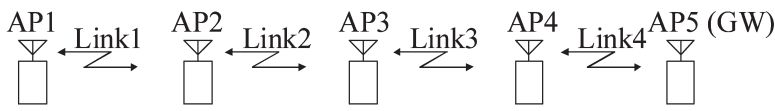

Fig. 14 Experimental configuration.

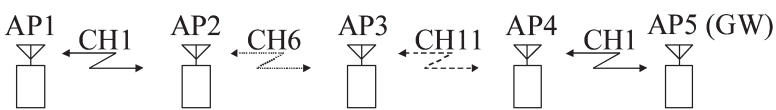

Fig. 15 Experimental results for channel allocation.

was $13 \mathrm{ch}$. The available channels were set to be 1,6 , and $11 \mathrm{ch}$. The gateway was the AP5 in Fig. 14.

Figure 15 denotes the channels for each link that were assigned by the proposed channel-allocation scheme using the configuration in Fig. 14. As can be seen in Figs. 14 and 15, Links 3 and 4 use different channels from Links 1 and 2. Here, the interference in Links 1 and 2 is Links 3 and 4. A simple network configuration of only five APs was established without STAs in this experiment to clarify the basic characteristics of the proposed antenna configuration and channel-allocation scheme. This experimental configuration is different from that in Fig. 13 and is a very simple 1-dimensional model. However, the main point in the channel-allocation scheme is that APs select different channels in neighboring links to establish a link. Therefore, the 


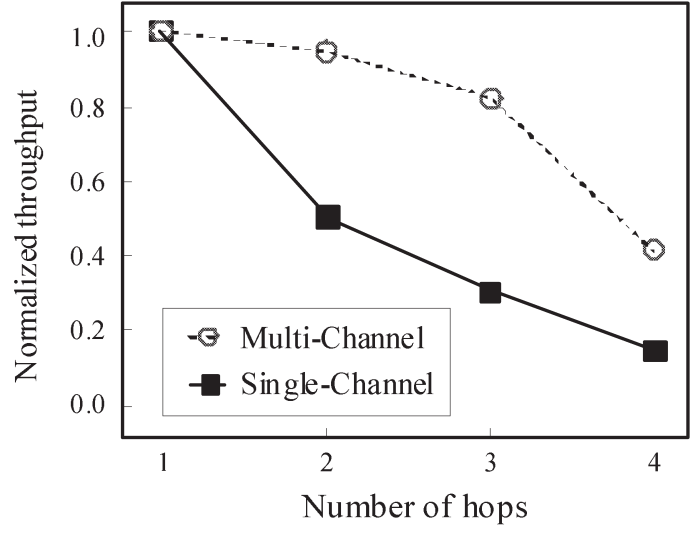

Fig. 16 Experimental results for channel allocation.

proposed scheme will still be effective even if it is carried out with the configuration in Fig. 13.

Next, we measured the UDP throughput for configurations of single and multi-channels using Netperf [21]. The physical distance between APs was set to $10 \mathrm{~m}$ and the sending pattern for UDP packets was set to CBR. The offered traffic was set to $40 \mathrm{Mb} / \mathrm{s}$ to fill the channel capacity of IEEE $802.11 \mathrm{~g}$ (physical data rate of $54 \mathrm{Mb} / \mathrm{s}$ ). The UDP packets were 1472 bytes. We measured the UDP throughput for the links of AP1-AP2 (1 hop), AP1-AP3 (2 hops), AP1-AP4 (3 hops), and AP1-AP5 (4 hops). Figure 16 denotes the normalized UDP throughput versus the number of hops. We set 1 as the value of throughput for the link from AP1 to AP2. As can be seen in this figure, the normalized throughput is $1 / N$ when the number of hops is $N$ for the single-channel configuration.

The normalized throughput, on the other hand, is much greater than $1 / N$ when the number of hops is $N$ for the multichannel configuration. Hence, we found that the multichannel configuration achieved higher throughput than that for the single channel. The isolation between antennas, the processing performance of the test bed, and the transfer delay in multi-hops were assumed to cause the slightly degraded throughput for 2 and 3 hops. For example, the influence of interference cannot completely be removed from the proposed antenna configuration, because slight mutual coupling (from about -40 to $-50 \mathrm{~dB}$ ) between antennas occurred, as shown in Fig. 10. Although the throughput for the 4th hop is low for the multi-channel configuration due to interference between Links 1 and 4 in Fig. 16, we consider such problems can be solved by combining the use of $5 \mathrm{GHz}$ band in IEEE802.11a. A basic experimental evaluation was employed by using a simple network to clarify the key characteristics of both the proposed configuration for antennas and allocation of channels that is implemented in an actual device. The experimental results from our ad-hoc system suggested that reliable operation could be obtained. However, more detailed evaluations are required when the proposed system in this paper is replaced in practice for large-scale network services. Hence, we intend to simulate a large-scale model in future work.

\section{Conclusion}

We proposed an ad-hoc relay transmission system with multiple-relay APs and channels to avoid the effects of interference due to the hidden-terminal problem and multiple APs inside a single piece of equipment. Moreover, we developed a testbed to evaluate the schemes we propose. The new system has two main features: 1) the suppression of interference from adjacent channels by using antennaradiation patterns with a vertical arrangement of antennas and 2) a distributed scheme for channel allocation which recognizes simple network topologies and allocates channels that are the most suited to the ad-hoc network.

We clarified how effective the proposed scheme was by using a testbed we developed. We confirmed that 1) an antenna configuration with a vertical antenna array could sufficiently reduce interference among APs inside a single piece of equipment, and 2) the proposed distributed allocation of channels could automatically avoid channels with interfering links due to the hidden-terminal problem. Moreover, it is confirmed that transmission was effective using multichannels by measuring UDP throughput to compare it to that using a single channel.

\section{References}

[1] IEEE Std 802.11, Part 11: Wireless LAN Medium Access. Control (MAC) and Physical Layer (PHY) Specifications, ISO/IEC 8802-11, 2008.

[2] L. Loyola, T. Kumagai, K. Nagata, S. Otsuki, and S. Aikawa, "A multi channel infrastructure based on DCF access mechanism for wireless LAN mesh networks compliant with IEEE 802.11," Proc. APCC2005, pp.497-501, Oct. 2005.

[3] C. Chiasserini and M. Meo, "An innovative routing scheme for 802.11- based multi-hop networks," Proc. VTC 2004-Fall, vol.4, pp.2804-2807, Sept. 2004.

[4] A. Yamada, A. Fujiwara, and Y. Matsumoto, "Enhancement of mesh network oriented IEEE 802.11 MAC protocol," Proc. APCC, vol.1, pp.142-146, Sept. 2004.

[5] IEEE802.11s Draft 3.02, Part 11: Wireless LAN Medium Access Control (MAC) and Physical Layer (PHY) specifications, Amendment 10: Mesh Networking, May 2009.

[6] IETF MANET(Mobile Ad-Hoc Networks), http://www.ietf.org/ html.charters/manet-charter.html

[7] C.C. Lo, C.Y. Kuo. Y.H. Huang, K.C. Lin, and Y.P. Chu, "Study on hidden receiver problem in mobile ad hoc networks," Proc. ICWMC 2008, pp.49-54, July 2008.

[8] M. Sekido, M. Takata, M. Bandai, and T. Watanabe, "A directional hidden terminal problem in ad hoc network MAC protocols with smart antennas and its solutions," Proc. IEEE Globecom 2005, vol.5 pp.2579-2583, Dec. 2005.

[9] D. Lihong and J. Yan'an, "A novel MAC protocol for hidden receiver problem in ad hoc networks," Proc. IEEE ICAL 2007, pp.23452348, Aug. 2007.

[10] K. Xu, M. Gerla, and S. Bae, "How effective is the IEEE802.11 RTS/CTS handshake in ad hoc networks?," Proc. IEEE ICC 2005, vol.1 pp.261-265, Dec. 2001.

[11] S. Xu and T. Saadawi, "Does the IEEE 802.11 MAC protocol work well in multihop wireless ad hoc networks?," IEEE Commun. Mag., vol., no., pp.130-137, June 2001.

[12] C.M. Cheng, P.H. Hsiao, H.T. Kung, and D. Vlah, "Adjacent channel 
interference in dual-radio 802.11a nodes and its impact on multihop networking," 2006 IEEE Global Telecommunications Conference (Globecom), Nov. 2006.

[13] M. Elhawary and Z.J. Haas, "Busy tone multi channel (BTMC): A new multi channel MAC protocol for ad hoc networks," Proc. IEEE PerCom 2008, pp.234-238, March 2008.

[14] J. LI, Z.J. Haas, M. Sheng, and Y. Chen, "Performance evaluation of modified IEEE 802.11 MAC for multi channel multi-hop ad hoc network," AINA'03, pp.312-317, 2003.

[15] J. Shi, T. Salonidis, and E.W. Knightly, "Starvation mitigation through multi channel coordination in CSMA multi-hop wireless networks," MobiHoc'06, May 2006.

[16] N. Kikuma, Adaptive Antenna Technologies, Ohmsha, 2003.

[17] K. Cho, K. Nishimori, Y. Takatori, and T. Hori, "Adaptive antennas employing vertical pattern control for street microcell," 1998 Proc. IEEE AP-S Symposium, vol.2, pp.639-642, June 1998.

[18] K. Nishimori and K. Cho, "A novel SDMA configuration using smart antenna adopting vertical pattern and polarization control," Proc. IEEE VTC 2003-Spring, vol.2, pp.871-875, April 2003.

[19] A. Raniwala, K. Gopalan, and T. Chiueh, "Centralized channel assignment and routing algorithms for multi channel wireless mesh networks," ACM SIGMOBILE Mobile Computing and Communications Review, vol.8, no.2, pp.50-65, April 2004.

[20] A.M. Das, H. Pucha, D. Koutsonikolas, Y.C. Hu, and D. Peroulis, "DMesh: Incorporating practical directional antennas in multichannel wireless mesh networks," IEEE J. Sel. Areas Commun., vol.24 no.11, pp.2028-2039, Nov. 2006.

[21] Netperf, http://www.netperf.org/

\section{Appendix: Time Spent in Allocating Channels}

The appendix explains the time spent by the system we developed in allocating channels. To evaluate the analytical time in the environment in Fig. 14, let the transmission time for an AP information request be Trequest and an AP information response be Tresponse, which are including overhead. For simplicity, we will evaluate the analytical time for 1 hop, 2 hops, 3 hops, and 4 hops. For example, AP4 and AP5 in Fig. 14 are only present for 1 hop, and AP3, AP4, and AP5 are only present for 2 hops. For 1 hop, since AP4 wants to allocate a channel to communicate with the gateway (AP5), AP4 broadcasts an AP information request and then AP5 transmits an AP information response. AP4 can allocate the channel through this frame exchange. Hence, the analytical time is Trequest + Tresponse for 1 hop. Since AP selects the links by the number of hops from the gateway and allocates a channel on the links as stated in Sects. 4.3 and 4.4, AP3 allocates the channel after AP4 has finished allocating the channel for 2 hops. After AP4 has broadcast an AP information request, AP3 and AP5 transmit an $\mathrm{AP}$ information response. Considering the analytical time for AP3, Trequest + Tresponse, the analytical time for 2 hops is Trequest $+2 \times$ Tresponse + Trequest + Tresponse $=$ $2 \times$ Trequest $+3 \times$ Tresponse. The analytical time in the other hops is calculated similarly. The analytical times are $1.6 \mathrm{~ms}$ for 1 hop, $4.3 \mathrm{~ms}$ for 2 hops, $6.9 \mathrm{~ms}$ for 3 hops, and $9.6 \mathrm{~ms}$ for 4 hops. For 4 hops, the AP1 farthest from the gateway takes the longest to finish operation, because information concerning the gateway is recognized last.

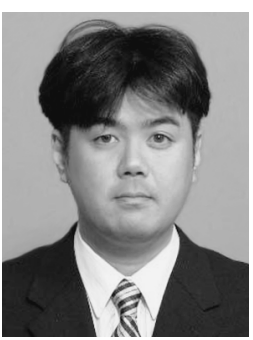

Kentaro Nishimori received the B.E., M.E. and Dr.Eng. degrees in electrical and computer engineering form Nagoya Institute of Technology, Nagoya, Japan in 1994, 1996 and 2002, respectively. In 1996, he joined the NTT Wireless Systems Laboratories, Nippon Telegraph and Telephone Corporation (NTT), in Japan. He was senior research engineer on NTT Network Innovation Laboratories. He is now associate professor in Niigata University. He was a visiting researcher at the Center for Teleinfrastructure (CTIF), Aalborg University, Aalborg, Denmark in 2006. He was an editor for the Transactions on Communications for the IEICE Communications Society and Assistant Secretary of Technical Committee on Antennas and Propagation of IEICE. He received the Young Engineers Award from the IEICE of Japan in 2001, Young Engineer Award from IEEE AP-S Japan Chapter in 2001, Best Paper Award of Software Radio Society in 2007 and Distinguished Service Award from the IEICE Communications Society in 2005 and 2008. His current research interest is Multi-user MIMO systems and cognitive radio systems. He is a member of IEEE.

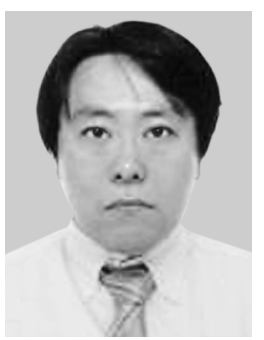

Takefumi Hiraguri received the M.E. and Ph.D. degrees from the University of Tsukuba, Ibaraki, Japan, in 1999 and 2008, respectively. In 1999, he joined the NTT Access Network Service Systems Laboratories, Nippon Telegraph and Telephone (NTT) Corporation. He is now associate professor in Nippon Institute of Technology. He has been engaged in research and development of high speed and high communication quality wireless LANs systems. $\mathrm{He}$ is a member of IEEE.

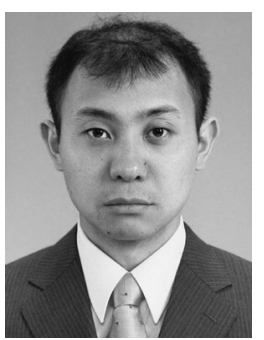

Masakatsu Ogawa received the B.E., M.E. and Ph.D. degrees from Sophia University, Tokyo, Japan, in 1998, 2000 and 2003, respectively. He was an adjunct member at National Institute of Informatics from 2003 to 2004, and a visiting researcher at Sophia University from 2003 to 2005. In 2004, he joined NTT Access Network Service Systems Laboratories, NTT Corporation. From 2004 to 2009, he was engaged in research and development of high speed wireless LANs. In 2009, he moved to the Technical Assistance and Support Center, NTT East Corporation. He received the IEICE Young Researcher's Award in 2007, and the IEICE Communications Society Distinguished Contributions Award in 2005, 2008 and 2009. He is a member of IEEE.

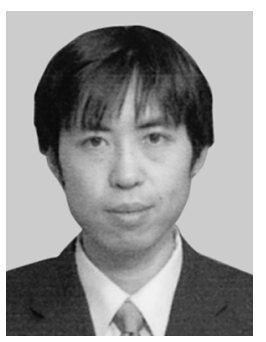

Naoki Honma received the B.E., M.E., and $\mathrm{Ph} . \mathrm{D}$. degrees in electrical engineering from Tohoku University, Sendai, Japan in 1996, 1998, and 2005, respectively. In 1998, he joined the NTT Radio Communication Systems Laboratories, Nippon Telegraph and Telephone Corporation (NTT), in Japan. He is now associate professor in Iwate University. He received the Young Engineers Award from the IEICE of Japan in 2003, the APMC Best Paper Award in 2003, and the Best Paper Award of IEICE Communication Society in 2006, respectively. His current research interest is planar antennas for high-speed wireless communication systems. He is a member of IEEE. 